\title{
Femtosecond imaging of surface plasmon dynamics in a nano-structured silver film
}

Atsushi Kubo, Ken Onda, Hrvoje Petek, Zhijun Sun, Yun S. Jung, Hong Koo Kim

(DOI: $10.1021 / \mathrm{n} 10506655)$

Caption of movie files of 'Supporting Information'.

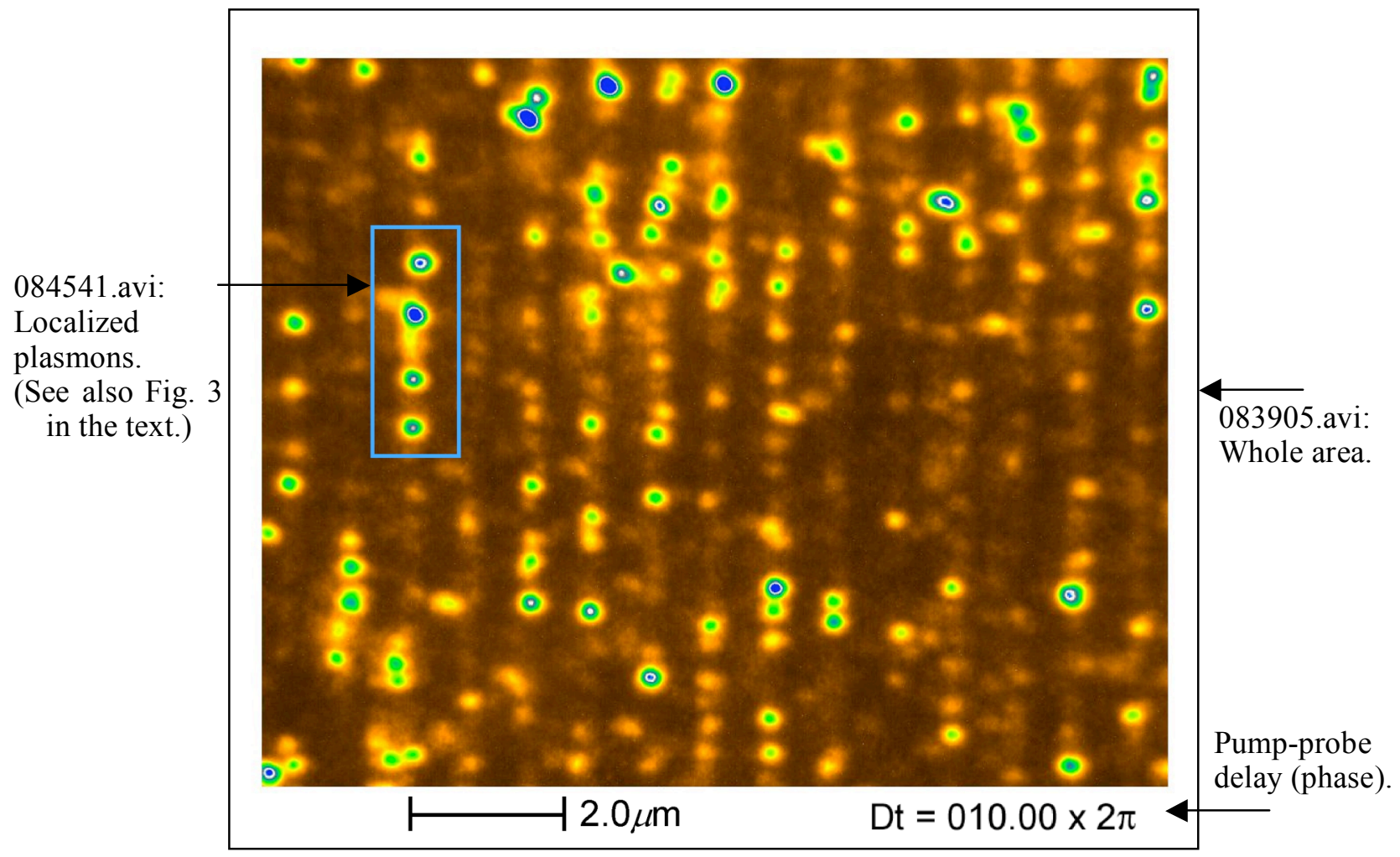

083905.avi. The movie of surface plasmon dynamics in a silver grating recorded with attosecond precision. The sample is irradiated by femtosecond phase-locked pulse pairs, and emitted photoelectrons are imaged by a photoelectron emission microscope (PEEM). The frame rate of 330 as, or 1/4 optical cycle of the carrier wave, corresponds to the interval by which the delay between identical pump and probe pulses is advanced. Movie 1 shows a field of view of $11.5 \times 9.2 \mu \mathrm{m}$. The range of pump-probe delays, $\tau_{d}$, is $9 \frac{3}{4} \sim$ $35 \frac{1}{2}$ optical cycles, which corresponds to 13.00 to $47.36 \mathrm{fs}$. At the beginning $\left(\tau_{d}<15 \times 2 \pi\right)$, the entire image oscillates in-phase due to optical interference between pump and probe pulses. After the pump pulse has passed, each dot starts to oscillate at its natural frequency. This results in a phase slip between the dots, so that their oscillations are no longer correlated at delays of $15 \times 2 \pi<\tau_{d}<25 \times 2 \pi$. At longer delays $\left(\tau_{d}>30 \times 2 \pi\right)$, the dot oscillations diminish due to polarization dephasing, and the intensity decays monotonically reflecting the phase independent population decay of hot electrons.

084541.avi. Magnified movie of the four localized plasmons modes indicated by the rectangle. Out-of-phase oscillations can be clearly seen in the intermediate delay range. Typical snapshots are extracted in Fig. 3 and their dynamics are discussed in the text. 